\title{
Novel peptide screened from a phage display library antagonizes the activity of $\mathrm{CC}$ chemokine receptor 9
}

\author{
YI HU $^{1 *}$, AIPING MA ${ }^{2 *}$, SHAN LIN ${ }^{3}$, YANG YANG $^{4}$ and GUOLIN HONG ${ }^{1}$ \\ Departments of ${ }^{1}$ Laboratory Medicine,${ }^{2}$ Respiratory Medicine and ${ }^{3}$ Orthopedics, \\ The First Affiliated Hospital of Xiamen University, Xiamen, Fujian 361003; ${ }^{4}$ College of Food and Biological Engineering, \\ Jimei University, Xiamen, Fujian 361021, P.R. China
}

Received September 29, 2016; Accepted August 8, 2017

DOI: $10.3892 / \mathrm{ol} .2017 .7065$

\begin{abstract}
CC chemokine receptor 9 (CCR9) serves a role in the drug resistance and metastasis of tumors. In the present study, a peptide specifically bound to CCR9 was obtained and the effect on tumor cells was observed. A Ph.D.-12 phage display peptide library was used to screen for peptides binding specifically to the second extracellular loop of CCR9. The ratios of the input and output of phage clones increased gradually following three rounds of biopanning. A total of 8 positive phage clones were identified from DNA analysis. A phage clone, C-4, was identified which exhibited higher affinity and specificity for the second extracellular loop of CCR9 in vitro compared with other clones. A peptide (P1; VHWDFRQWWQPS) was identified which may inhibit the corresponding phage, $\mathrm{C}-4$, binding to the second extracellular loop of CCR9. Furthermore, P1 was able to bind specifically with MOLT4 cells which exhibit marked expression of CCR9. In addition, P1 promoted the apoptosis of MOLT4 cells induced by doxorubicin, and inhibited the migration of MOLT4 cells in the presence of chemokine (C-C motif) ligand 25 . It was suggested that decreased activity in the phosphorylation of protein kinase B in MOLT4 cells may be responsible for the inhibition. In conclusion, the peptide P1 derived from a screened phage is able to specifically bind to CCR9 and inhibit the activity of CCR9. It has potential use as an antagonist in the treatment of CCR9-overexpressed carcinoma.
\end{abstract}

\section{Introduction}

CCR9 is a member of the family of G-protein-coupled receptors (GPCRs) and serves a role in T-cell differentiation

Correspondence to: Professor Guolin Hong, Department of Laboratory Medicine, The First Affiliated Hospital of Xiamen University, 55 Zhenhai Road, Xiamen, Fujian 361003, P.R. China E-mail: 18860089899@139.com

${ }^{*}$ Contributed equally

Key words: CC chemokine receptor 9, peptide, tumor, antagonist, apoptosis, migration and tissue-specific homing once bound to its cognate ligand chemokine (C-C motif) ligand 25 (CCL25) (1). CCR9 has also been implicated in numerous types of cancer including breast, lung, pancreas, prostate and colon cancer, in addition to adult T-cell leukemia; it is also reported that CCR9 mediates organ-selective metastasis and drug resistance (2-7). The functions of CCR9 in metastasis and drug resistance depend on the activation of the phosphoinositide 3-kinase $(\mathrm{PI} 3 \mathrm{~K}) /$ protein kinase $\mathrm{B}$ (Akt) signaling pathway (8). Furthermore, the differences in the cell-surface profile between cancer cells and their normal counterparts may be used as a molecular signature for targeted therapy and drug delivery. Thus, CCR 9 is a promising candidate for a target molecule based on its rare cell-surface expression in normal tissues and increased cell-surface expression in malignancies.

An immunotoxin CCL25-PE38 has previously been developed that is able to specifically kill high CCR9-expressing MOLT4 cells (9). However, several issues remain unresolved, including uptake and expression of CCL25-PE38. Peptides exhibit a short plasma half-life, increased tissue penetration ability, decreased immunogenicity, and are easily synthesized in comparison with macromolecular proteins (10).

Phage display is a molecular technology that permits the presentation of a number of peptides with high specificity and affinity for a target on the surface of a bacteriophage. For instance, the biopanning of phage display libraries on cellular receptors has been successful in selecting peptides that exhibit high affinity and specificity for the receptors (11-13). Therefore, peptides screened from phage display may be useful for the diagnosis and targeted therapy of tumors.

In the present study, the second extracellular loop of CCR9 was used to screen a 12-mer phage display library and a novel peptide (P1; VHWDFRQWWQPS) that was capable of specifically binding to CCR9 was identified. Furthermore, this peptide promoted the apoptosis of MOLT4 cells induced by doxorubicin (DOX) and inhibited the migration of MOLT4 cells in the presence of CCL25. The inhibitory effects may be associated with inhibition of the activation of Akt in MOLT4 cells. In conclusion, the novel peptide identified in the present study may inhibit the activity of CCR9 and is potentially useful in the research of novel treatments for CCR9-overexpressed carcinoma. 


\section{Materials and methods}

Affinity screening procedure. The Ph.D.-12 phage display library (version 1.1; New England BioLabs, Ipswich, MA, USA) was used as the biopanning tool. The panning procedure was performed according to the manufacturer's protocol. Briefly, the second extracellular loop of CCR9 (AAADQWKFQTFMCKVVNSMYK; SBS Genetech Co., Ltd., Beijing, China) was used to coat microtiter plates at $10 \mu \mathrm{g} / \mathrm{ml}$. Phages that specifically bound to the target were identified through three rounds of in vitro selection. In each round, the bound phages were rescued and amplified in ER2738 bacterial cells from the phage display library (New England BioLabs, Inc., Ipswich, MA, USA) for the subsequent panning, whereas the unbound phages were removed by washing with Tris-buffered saline with Tween-20 (TBST). The washing conditions for the second and third rounds were the same as those for the first round, with the exception that the Tween-20 concentration was increased from 0.1 to $0.5 \%$, respectively. Following the elution step in the third round, the single clones of phage particles were harvested. The phages were subjected to DNA extraction, sequencing and an ELISA.

DNA sequencing of the selected phages. Following the third round of panning, 16 phage clones were randomly selected, amplified and purified. The clones were designated C-1 to C-16. Single-stranded DNA was extracted with M13 DNA Kit (Beijing BLKW Biotechnology Co., Ltd., Beijing, China) according to the manufacturer's protocol. DNA sequencing was performed by Sangon Biotech Co., Ltd. (Shanghai, China) using the $-96 \mathrm{gIII}$ primer (5'-CCCTCATAGTTAGCGTAA CG-3'). Sequence analysis was performed and phages with the same sequence were classified.

Phage capture ELISA. A 10- $\mu \mathrm{g} / \mathrm{ml}$ sample of the second extracellular loop of CCR9 in $0.1 \mathrm{M} \mathrm{NaHCO}_{3}$, pH 8.6, was coated on ELISA plates. The ELISA plates were incubated overnight at $4^{\circ} \mathrm{C}$ in an air-tight humidified box. The excess solution was removed and the plates were blocked with a blocking buffer $\left(0.1 \mathrm{M} \mathrm{NaHCO}_{3}(\mathrm{pH} 8.6), 5 \mathrm{mg} / \mathrm{ml} \mathrm{BSA}\right)$ for $1 \mathrm{~h}$ at $4^{\circ} \mathrm{C}$. Subsequently, the plates were washed and phages including control phage, C-4, C-6, C-9 and C-10 [at $10^{11}$ plaque-forming units (pfu)] were added to the target-coated wells. The plates were incubated at room temperature for $1 \mathrm{~h}$ with agitation. Finally, a horseradish peroxidase-conjugated anti-M13 monoclonal antibody at $5 \mu \mathrm{g} / \mathrm{ml}$ (cat. no. 111973-MM05; Sino Biological, Inc., Beijing, China) was used to detect the bound phages. Data are reported from three independent experiments.

Peptide synthesis and labeling. The candidate peptide P1 (VHWDFRQWWQPS) and unrelated peptide (control P; WIRPPSGPMYSF) were synthesized by SBS Genetech, Co., Ltd. and Sangon Biotech Co., Ltd., respectively, using standard solid-phase chemistry. Fluorescein isothiocyanate (FITC) was conjugated to the N-terminus of P1 (FITC-P1) or the unrelated peptide (FITC-control P). The products were purified to a minimum purity of $95 \%$ using high-performance liquid chromatography and isolated by lyophilization. The sequence and structure of each peptide were characterized by mass spectrometry.
Competitive inhibition assay. To investigate the inhibition effect of P1 on C-4, a competitive inhibition ELISA was conducted. Briefly, wells were incubated with $10 \mu \mathrm{g} / \mathrm{ml}$ of the second extracellular loop of CCR9. P1 at various concentrations $(0,0.01,0.1,0.5,1.0,2.0$ and $10 \mathrm{nmol} / \mathrm{ml})$ was added to the cells. Subsequently, $10^{11} \mathrm{pfu}$ C-4 was added to the wells prior to incubation at $37^{\circ} \mathrm{C}$ for $1 \mathrm{~h}$. The subsequent procedure was similar to that of the ELISA performed for the aforementioned phage capture. The wells were washed with 1x PBS (pH 7.4) 3 times following incubation. Finally, a horseradish peroxidase-conjugated anti-M13 monoclonal antibody at $10 \mu \mathrm{g} / \mathrm{ml}$ (cat. no. 111973-MM05; Sino Biological, Inc.) was used to detect the bound phages. The rate of inhibition was calculated using the following formula: Rate of inhibition=[optical density (OD) of control P-OD of P1]/OD of control P x 100\%. An unrelated peptide was used as the control P. Data are reported from three independent experiments.

Confocal microscopy analysis. MOLT4 cells were obtained from the American Type Culture Collection (ATCC; Manassas, VA, USA). Cells were maintained in RPMI-1640 medium (GE Healthcare Life Sciences, Logan, UT, USA) supplemented with $10 \%$ fetal bovine serum (GE Healthcare Life Sciences), $100 \mathrm{U} / \mathrm{ml}$ penicillin and $100 \mathrm{mg} / \mathrm{ml}$ streptomycin. The cells were cultured at $37^{\circ} \mathrm{C}$ in a humidified atmosphere containing $5 \% \mathrm{CO}_{2}$. The MOLT4 cells were washed three times with PBS. One group was washed three times with PBS and blocked with $2 \%$ bovine serum albumin (BSA; Santa Cruz Biotechnology, Inc., Dallas, TX, USA) at $4^{\circ} \mathrm{C}$ for $30 \mathrm{~min}$ and FITC-P1 $(0.1 \mathrm{nmol} / \mathrm{ml})$ was incubated with the cells for $1 \mathrm{~h}$ at $4^{\circ} \mathrm{C}$. Meanwhile, another group was incubated with anti-CCR9 monoclonal antibody at $0.1 \mathrm{nmol} / \mathrm{ml}$ (anti-CCR $9 \mathrm{mAb}$; cat. no. 112509; R\&D Systems, Inc., Minneapolis, MN, USA) for $30 \mathrm{~min}$ at $37^{\circ} \mathrm{C}$, blocked with $2 \%$ BSA and subsequently incubated with FITC-P1 at $4^{\circ} \mathrm{C}$ as above. Following three washes, the cells were placed on poly-L-lysine-coated slides and observed using a laser-scanning confocal microscope with the magnification, x200 (LSM710; Zeiss GmbH, Jena, Germany). FITC-control P was used as the control.

Apoptosis assay. MOLT4 cells (4x10\%/well) were transferred to 24-well plates with each well containing $500 \mu$ l RPMI-1640 medium without FBS. Each well was treated with a distinct concentration of $\mathrm{P} 1(0,0.1$ and $1.0 \mathrm{nmol} / \mathrm{ml})$ for $12 \mathrm{~h}$, and then treated with DOX $(1 \mu \mathrm{g} / \mathrm{ml})$ for $12 \mathrm{~h}$. The control groups were treated with various concentrations of P1 $(0,0.1$ and $1.0 \mathrm{nmol} / \mathrm{ml}$ ) for $24 \mathrm{~h}$. The cells were washed twice, and subsequently detected using an Annexin V-FITC and propidium iodide (PI) double staining kit (Invitrogen; Thermo Fisher Scientific, Inc., Waltham, MA, USA) for $15 \mathrm{~min}$ at $25^{\circ} \mathrm{C}$ in the dark, according to the manufacturer's protocol. Subsequently, the processed cells were rinsed with PBS and subjected to fluorescence-activated cell sorting (FACS) analysis. RBL-2H3 cells (ATCC, Manassas, VA, USA), were incubated with Dulbecco's modified Eagle's medium (Thermo Fisher Scientific, Inc.) at $4 \times 10^{5}$ cells/well. The subsequent procedure was the same as that for the MOLT4 cells. Results were presented as the percentage of Annexin V-FITC-positive cells, Annexin V-FITC- and PI-double-positive cells. Data are reported from three independent experiments. 
Table I. Enrichment of phages screened from the Ph.D.-12 library.

Titer, pfu

\begin{tabular}{|c|c|c|c|c|}
\hline \multirow[b]{2}{*}{ Round } & \multirow{2}{*}{$\begin{array}{c}\text { Second extracellular } \\
\text { loop of CCR } 9, \mu \mathrm{g}\end{array}$} & \\
\hline & & Input & Output & Recovery \\
\hline First & 10 & $1.0 \times 10^{11}$ & $5.2 \times 10^{5}$ & $5.2 \times 10^{-6}$ \\
\hline Second & 5 & $1.0 \times 10^{11}$ & $3.6 \times 10^{6}$ & $3.3 \times 10^{-5}$ \\
\hline Third & 2 & $1.2 \times 10^{9}$ & $4.9 \times 10^{5}$ & $4.1 \times 10^{-4}$ \\
\hline
\end{tabular}

The output/input ratio was used to indicate the recovery of phages. CCR9, CC chemokine receptor 9; pfu, plaque-forming units.

Chemotaxis assay. The chemotaxis assay was performed in 24-well Transwell chambers (Corning Life Sciences, Cambridge, MA, USA) as previously described (14). MOLT4 cells $\left(0.5 \times 10^{4}\right.$ cells/well) were treated with $\mathrm{P} 1(0,0.1$ and $1.0 \mathrm{nmol} / \mathrm{ml})$ and control $\mathrm{P}(0,0.1$ and $1.0 \mathrm{nmol} / \mathrm{ml})$ for $1 \mathrm{~h}$, prior to being added to the upper chamber. CCL25 $(0.1 \mathrm{nmol} /$ $\mathrm{ml})$ was placed in the lower chamber $(500 \mu \mathrm{l})$. Following incubation at $37^{\circ} \mathrm{C}$ in $5 \% \mathrm{CO}_{2}$ for $2 \mathrm{~h}$, cells were collected from the lower wells. The number of cells was counted using light microscopy. Spontaneous baseline migration (BM) was used as a control. The experiments were performed in triplicate.

Western blotting. To investigate the expression levels of Akt and phospho-Akt ( $\mathrm{p}-\mathrm{Akt}$ ) in MOLT4 cells in the presence of P1, MOLT4 cells incubated in 24-well plates were serum-starved for $12 \mathrm{~h}$ and then treated with $\mathrm{P} 1$ at various concentrations $(0,0.1$ and $1.0 \mathrm{nmol} / \mathrm{ml})$ for $24 \mathrm{~h}$. The protein extraction procedure was performed as described previously (15). The nitrocellulose membranes were incubated overnight at $4^{\circ} \mathrm{C}$ with anti-p-Akt $\left(\operatorname{Ser}^{473}\right)$ at 1:100 (cat. no. 9271; Cell Signaling Technology, Danvers, MA, USA) or anti-Akt monoclonal antibody at 1:100 (cat. no. 9272, Cell Signaling Technology) and incubated with horseradish peroxidase-conjugated secondary antibody at 1:2,000 (cat. no. D110058-0100; Sangon Biotech Co., Ltd.). $\beta$-actin was used as the control. Finally, the membrane-bound proteins were detected using enhanced chemiluminescence substrate (Pierce ECL; cat. no. 32209 , Thermo Fisher Scientific, Inc.). The blots were incubated with the ECL working solution for $1 \mathrm{~min}$ at room temperature, and then removed and placed in a film cassette with the protein side facing up in the dark for X-ray film exposure. The film was developed using the developing solution (cat. no., AR0132; BOSTER Biological Technology Co., Ltd, Wuhan, China) for $2 \mathrm{~min}$ and fixative solution (cat. no. AR0132; BOSTER Biological Technology Co., Ltd, Wuhan, China) for $1 \mathrm{~min}$ at room temperature following exposure of $60 \mathrm{sec}$. The experiments were performed in triplicate.

Statistical analysis. The data are expressed as the mean \pm standard deviation or a percentage. Data analysis was performed using GraphPad Prism (Version 5; GraphPad Software, Inc., La Jolla, CA, USA). One-way analysis of variance and Student's t-test were used to perform statistical comparisons. $\mathrm{P}<0.05$ was considered to indicate a statistically significant difference.

\section{Results}

Screening of the Ph.D.-12 phage display library against CCR9. To obtain a specific peptide binding to the CCR9, a Ph.D.-12 phage display library was screened for binding to the second extracellular loop of CCR9. Following three rounds of biopanning, the titers of phages increased $~ 79$-fold compared with the titer in the first round. The output/input ratio of phages following each round of panning was used to determine the enrichment efficiency, which increased from $5.2 \times 10^{-6}$ to $2.4 \times 10^{-4}$ (Table I). This increase indicated that phages capable of specifically binding to the second extracellular loop of CCR9 were significantly enriched following the biopanning.

DNA sequencing of the selected phage clones. The results demonstrated that 8 phage clones lacked an exogenous sequence and the remaining 8 clones were confirmed to be positive using DNA sequencing. A total of 8 phage clones and corresponding peptide sequences were obtained (Table II). Sequence alignment analysis did not identify homology among the peptide sequences.

Phage-binding assay. An ELISA was performed to determine the affinity and specificity of positive phage clones. The results revealed that the phage $\mathrm{C}-4$ appeared to exhibit increased affinity for the target molecule in comparison with the other phages (Fig. 1). The affinity of C-4 increased $>8$-fold compared with the control phage, a statistically significant increase $(\mathrm{P}<0.01)$. It also identified a significant difference between group $\mathrm{C}-4$ and group $\mathrm{C}-6(\mathrm{P}<0.05), \mathrm{C}-9$ $(\mathrm{P}<0.05)$ and $\mathrm{C}-10(\mathrm{P}<0.05)$. Therefore, the displaying peptide (VHWDFRQWWQPS) derived from C-4 was designated P1 and was investigated further.

Competitive inhibition assay. To investigate whether the synthetic peptide $\mathrm{P} 1$ was able to inhibit the corresponding phage binding to the target molecule, competitive inhibition ELISA was performed. As presented in Fig. 2, C-4 binding to the target decreased following an increase in peptide P1. The peptide P1 appeared to inhibit C-4 binding to the second extracellular loop of CCR9 in a dose-dependent manner. When the concentration of $\mathrm{P} 1 \mathrm{reached} 1.0 \mathrm{nmol} / \mathrm{ml}, \mathrm{C}-4$ binding to the second extracellular loop of CCR9 was significantly inhibited (inhibition of 50.33\%).

Identification of Pl binding specifically to CCR9high-expressing cells. To investigate the peptide specifically binding to CCR9, 
Table II. DNA sequences of the selected phage clones.

\begin{tabular}{llc}
\hline Phage clone & \multicolumn{1}{c}{ DNA sequence } & Peptide sequence \\
\hline C-1/-2/-4/13/-14 & GTGCATTGGGATTTTCGG & VHWDFRQWWQPS \\
& CAGTGGTGGCAGCCTTCT & \\
C-6 & GGGGATGGTAATTCGGTG & GDGNSVLKPGNW \\
& CTGAAGCCGGGGAATTGG & \\
C-9 & CATCTTAGTCTTCCGCTTTG & HLSLPLWKWEKS \\
C-10 & GAAGTGGGAGAAGTCG & \\
& GTGTTTGCTTATCATCTT & VFAYHLRIPSGD \\
& AGGATTCCTTCTGGGGAT & \\
\hline
\end{tabular}

A total of 8 clones were identified to be endowed with four types of exogenous sequences following the third round of panning. The number of positive clones is presented as the frequency.

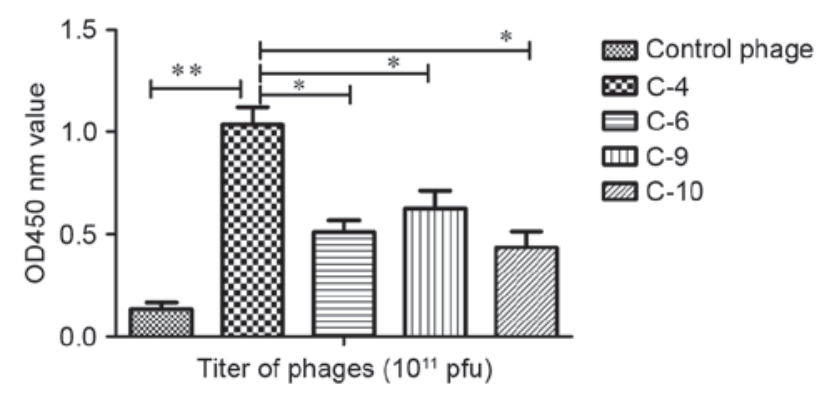

Figure 1. Identification of specific binding of phage clones to the second extracellular loop of $\mathrm{CC}$ chemokine receptor 9 . Affinity was detected using ELISA. The affinity of C- 4 increased $>8$-fold compared with the control phage, and was increased compared with the other phage clones $\left({ }^{*} \mathrm{P}<0.05\right.$; $\left.{ }^{* *} \mathrm{P}<0.01\right)$. OD, optical density; pfu, plaque-forming units.

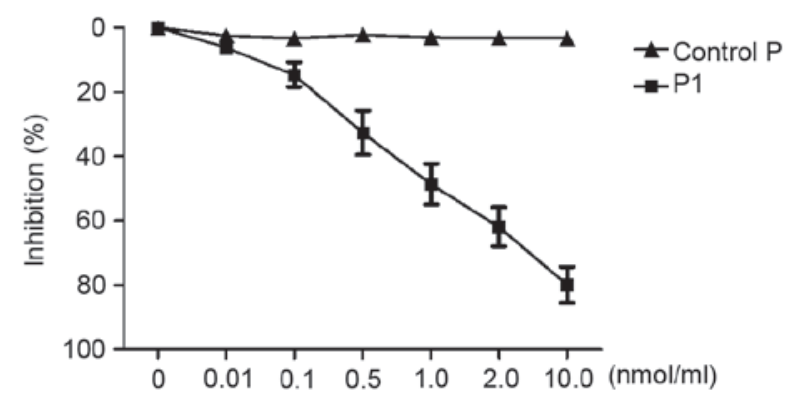

Figure 2. Inhibition of C-4 binding by $\mathrm{P} 1$. The binding of the positive phage $\mathrm{C}-4$ to the target decreased following an increase in P1. When the concentration of $\mathrm{P} 1$ reached $1.0 \mathrm{nmol} / \mathrm{ml}$, inhibition was $>50.0 \%$. An unrelated peptide was used as control P. Data are reported from three independent experiments.

confocal microscopy analysis was performed to evaluate the binding specificity of the peptide P1. It was revealed that FITC-P1 bound directly to MOLT4 cells, and the anti-CCR9 $\mathrm{mAb}$ was able to completely block the binding of P1. When a control peptide was incubated with MOLT4 cells, no cell binding was observed (Fig. 3). This suggests that P1 is able to specifically bind to CCR9 and is stable under physiological conditions.

P1 increases apoptosis of MOLT4 cells induced by DOX. To investigate the effect of P1 on cell apoptosis, the number of
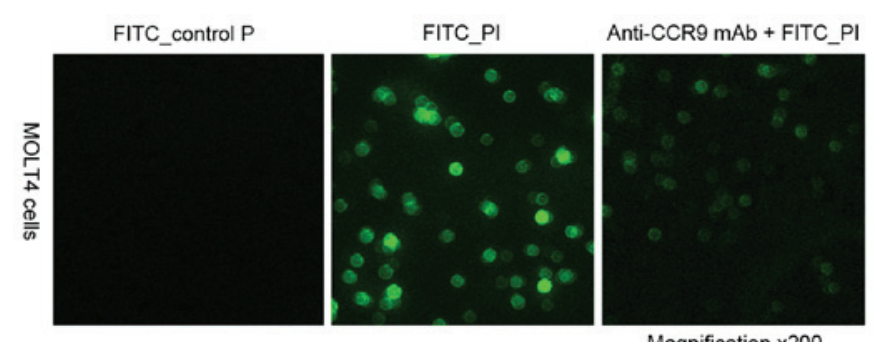

Figure 3. P1 binds specifically on cells which highly express CC chemokine receptor 9. FITC-control P (left panel) did not bind to MOLT4 cells; FITC-P1 (middle panel) did bind to MOLT4 cells; anti-CCR9 mAb (right panel) inhibited FITC-P1 binding to MOLT4 cells. Cells were observed using a laser-scanning confocal microscope; magnification, x200. CCR9, $\mathrm{CC}$ chemokine receptor 9; mAb, monoclonal antibody; FITC, fluorescein isothiocyanate; PI, propidium iodide.

apoptotic cells was measured using FACS following P1 and DOX treatments of MOLT4 cells and RBL-2H3 cells. As presented in Table III, the number of apoptotic MOLT4 cells induced by DOX increased to $64.7 \%$ following pre-treatment with $0.1 \mathrm{nmol} / \mathrm{ml} \mathrm{P1}(\mathrm{P}<0.05)$; apoptotic MOLT4 cells further increased to $79.5 \%$ following pre-treatment with $1.0 \mathrm{nmol} /$ $\mathrm{ml} \mathrm{P1}(\mathrm{P}<0.01)$. On the other hand, the number of apoptotic RBL-2H3 cells induced by DOX revealed no significant difference between cells treated with $\mathrm{P} 1$ and those receiving no treatment. These results suggest that $\mathrm{P} 1$ may elicit its effect on MOLT4 cells by promoting an apoptotic pathway.

P1 inhibits migration of MOLT4 cells. To evaluate the effects of P1 on migration of MOLT4 cells, a Boyden chamber migration assay was performed. As presented in Fig. 4, P1 significantly inhibited the migration of CCR9-expressing MOLT4 cells induced by CCL25 in a dose-dependent manner. No statistical comparison between the different doses was performed. In contrast, the control P did not affect the migration of MOLT4 cells induced by CCL25. A statistically significant difference between $\mathrm{P} 1$ and group control $\mathrm{P}$ was identified $(\mathrm{P}<0.05)$, revealing that $\mathrm{P} 1$ may antagonize the effect of CCL25.

Pl inhibits the phosphorylation of Akt in MOLT4 cells. As presented in Fig. 5, P1 caused a detectable inhibitory effect 
Table III. Effect of P1 on apoptosis induced by DOX.

A, MOLT4 cells

\begin{tabular}{lrcc}
\hline & \multicolumn{3}{c}{$\mathrm{P} 1, \mathrm{nmol} / \mathrm{ml}$} \\
\cline { 2 - 4 } Treatment & \multicolumn{1}{c}{0} & 0.1 & 1.0 \\
\hline Control & $5.3 \pm 0.6$ & $4.8 \pm 0.5$ & $6.1 \pm 1.0$ \\
DOX & $52.4 \pm 2.5$ & $64.7 \pm 3.1^{\mathrm{a}}$ & $79.5 \pm 4.2^{\mathrm{b}}$ \\
\hline
\end{tabular}

B, RBL-2H3 cells

$\mathrm{P} 1, \mathrm{nmol} / \mathrm{ml}$

\begin{tabular}{lrrr}
\cline { 2 - 4 } Treatment & 0 & \multicolumn{1}{c}{0.1} & \multicolumn{1}{c}{1.0} \\
\hline Control & $4.3 \pm 0.5$ & $6.6 \pm 0.8$ & $5.9 \pm 0.4$ \\
DOX & $50.1 \pm 3.0$ & $52.3 \pm 2.6$ & $51.5 \pm 2.9$ \\
\hline
\end{tabular}

The effect of P1 on cell apoptosis induced by DOX was determined using fluorescence-activated cell sorting. Values are percentages. (A) For MOLT4 cells, P1 significantly increased number of apoptotic cells induced by DOX, ${ }^{\mathrm{a}} \mathrm{P}<0.05(0.1 \mathrm{nmol} / \mathrm{ml} \mathrm{P} 1$ and $\mathrm{DOX}$ group vs. only DOX group), ${ }^{b} \mathrm{P}<0.01(1.0 \mathrm{nmol} / \mathrm{ml} \mathrm{P} 1$ and $\mathrm{DOX}$ group vs. only DOX group). (B) For RBL-2H3 cells, no significant difference between groups treated with P1 and DOX compared with only DOX was identified. The data are presented as the mean \pm standard deviation. The experiments were carried out three times. DOX, doxorubicin

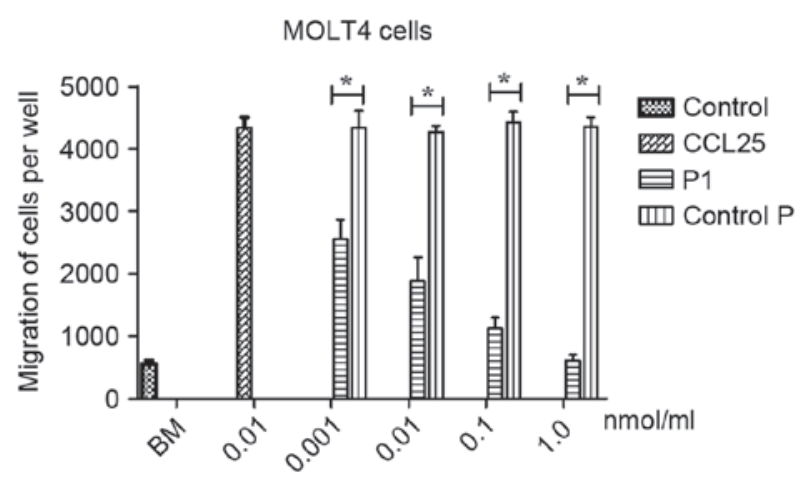

Figure 4. Chemotaxis of MOLT4 cells in the presence of P1. P1 may inhibit the migration of MOLT4 cells induced by CCL25. In contrast, control P had no effect on the migration of MOLT4 cells induced by CCL25 ( $\left.{ }^{*} \mathrm{P}<0.05\right)$. Spontaneous BM was used as a control; CCL25 was used as a positive control. BM, baseline migration.

on the expression of p-Akt in MOLT4 cells. The level of p-Akt was significantly different when compared with Akt as a control. Each dose of P1 was also significantly different from the subsequent dose, indicating that the effect of P1 is dose-dependent $(\mathrm{P}<0.05)$. This result implies that $\mathrm{P} 1$ may block the downstream CCR9 signaling pathway.

\section{Discussion}

Phage display has been proved to be an effective approach for screening the interaction of molecules, and to develop
A

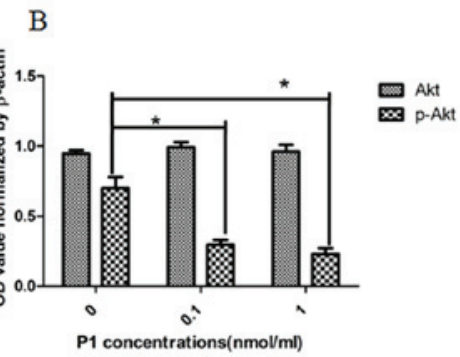

Figure 5. P1 inhibits the phosphorylation of Akt in MOLT4 cells. (A) The levels of Akt and p-Akt in MOLT4 cells were detected using western blot analysis following P1 treatment. (B) OD of Akt and p-Akt, normalized using $\beta$-actin. ${ }^{*} \mathrm{P}<0.05$. Akt, protein kinase $\mathrm{B}$; p-Akt, phospho-Akt; OD, optical density.

novel agents for the diagnosis and treatment of numerous types of cancer (16). In the present study, the second loop of CCR9 was used as the target molecule to screen the Ph.D.-12 phage display library for the first time, to the best of our knowledge. Following three rounds of biopanning, 8 positive phage clones expressing exogenous sequences were obtained. Of these positive clones, C-4 appeared five times and exhibited increased affinity compared with other phages detected by ELISA. Furthermore, the results revealed that P1 (VHWDFRQWWQPS) derived from C-4 exhibits specificity towards and affinity for the second extracellular loop of CCR9.

To investigate the ability of P1 binding to cells under physiological conditions, a fluorescently labeled peptide (FITC-P1) was synthesized and confocal microscopy analysis was performed. It was demonstrated that FITC-P1 may bind specifically to MOLT4 cells through CCR9. These results suggest that P1 may specifically bind to the second loop of CCR9.

CCR9 may serve a role in the drug resistance and metastasis of tumors. Furthermore, CCR9 may increase migration and invasion of tumor cells, and activate of PI3K/Akt signaling pathway in order to inhibit the apoptosis of tumor cells as induced by chemotherapeutic agents $(5,8)$. Experiments were performed to determine the effect of P1 on CCR9-overexpressing cells and the phosphorylation of Akt. As expected, the results indicate that P1 may increase the apoptosis of MOLT4 cells as induced by chemotherapeutic agents. Furthermore, P1 may inhibit the migration of CCR9-expressing MOLT4 cells induced by CCL25. Furthermore, it was revealed that P1 may significantly decrease phosphorylation of Akt. The results of the present study identified that $\mathrm{P} 1$ may have an effect on CCR9-overexpressing cells, increasing their susceptibility to chemotherapy and decreasing chemotaxis.

In conclusion, a novel peptide, P1 (VHWDFRQWWQPS), was developed which may inhibit the activity of CCR9. P1 may possess potential as a novel treatment for types of cancer which overexpress CCR9. However, further investigation into the use of P1 as an inhibitor for carcinoma, including the plasma half-life, tissue penetration ability, immunogenicity and in vivo antitumor effect is required.

\section{Acknowledgements}

This study was supported, in part, by the Natural Science Foundation of Fujian Province, China (grant no. 2013D012) and 
National Science Foundation of China (grant no. 81371902). The authors thank Mr. Fuyi Huang (Institute of Urban Environment, Chinese Academy of Sciences, Xiamen, China) for support with technology.

\section{References}

1. Zlotnik A and Yoshie O: Chemokines: A new classification system and their role in immunity. Immunity 12: 121-127, 2000.

2. Nagakubo D, Jin Z, Hieshima K, Nakayama T, Shirakawa AK, Tanaka Y, Hasegawa H, Hayashi T, Tsukasaki K, Yamada Y, et al: Expression of CCR9 in HTLV-1 ${ }^{+}$T cells and ATL cells expressing tax. Int J Cancer 120: 1591-1597, 2007.

3. Heinrich EL, Arrington AK, Ko ME, Luu C, Lee W, Lu J and Kim J: Paracrine activation of chemokine receptor CCR9 enhances the invasiveness of pancreatic cancer cells. Cancer Microenviron 6: 241-245, 2013.

4. Gupta P, Sharma PK, Mir H, Singh R, Singh N, Kloecker GH, Lillard JW Jr and Singh S: CCR9/CCL25 expression in non-small cell lung cancer correlates with aggressive disease and mediates key steps of metastasis. Oncotarget 5: 10170-10179, 2014.

5. Sharma PK, Singh R, Novakovic KR, Eaton JW, Grizzle WE and Singh S: CCR9 mediates PI3K/AKT-dependent antiapoptotic signals in prostate cancer cells and inhibition of CCR9-CCL25 interaction enhances the cytotoxic effects of etoposide. Int $\mathrm{J}$ Cancer 127: 2020-2030, 2010.

6. Singh S, Singh UP, Stiles JK, Grizzle WE and Lillard JW Jr: Expression and functional role of CCR9 in prostate cancer cell migration and invasion. Clin Cancer Res 10: 8743-8750, 2004.

7. Chen HJ,Edwards R, Tucci S, Bu P, Milsom J,Lee S, Edelmann W, Gumus ZH, Shen X and Lipkin S: Chemokine 25-induced signaling suppresses colon cancer invasion and metastasis. J Clin Invest 122: 3184-3196, 2012.
8. Tu Z, Xiao R, Xiong J, Tembo KM, Deng X, Xiong M, Liu P, Wang $M$ and Zhang Q: CCR9 in cancer: Oncogenic role and therapeutic targeting. J Hematol Oncol 9: 10, 2016.

9. Hu Y, Zhang L, Wu R, Han R, Jia Y, Jiang Z, Cheng M, Gan J, Tao X and Zhang Q: Specific killing of CCR9 high-expressing acute T lymphocytic leukemia cells by CCL25 fused with PE38 toxin. Leuk Res 35: 1254-1260, 2011.

10. Brasnjevic I,Steinbusch HW, Schmitz C and Martinez-Martinez P; European NanoBioPharmaceutics research initiative: Delivery of peptide and protein drugs over the blood-brain barrier. Prog Neurobiol 87: 212-251, 2009.

11. Wang FY, Zhang TY, Luo JX, He GA, Gu QL and Xiao F: Selection of CC chemokine receptor 5-binding peptide from a phage display peptide library. Biosci Biotechnol Biochem 70: 2035-2041, 2006.

12. Skelton NJ, Chen YM, Dubree N, Quan C, Jackson DY, Cochran A, Zobel K, Deshayes K, Baca M, Pisabarro MT, et al: Structure-function analysis of a phage display-derived peptide that binds to insulin-like growth factor binding protein 1 . Biochemistry 40: 8487-8498, 2001.

13. Tang B, Li Z, Huang D, Zheng L and Li Q: Screening of a specific peptide binding to VPAC1 receptor from a phage display peptide library. PLoS One 8: e54264, 2013.

14. Houimel M and Mazzucchelli L: Identification of biologically active peptides that inhibit binding of human CXCL8 to its receptors from a random phage-epitope library. J Leukoc Biol 85: 728-738, 2009.

15. Li B, Wang Z, Zhong Y, Lan J, Li X and Lin H: CCR9-CCL25 interaction suppresses apoptosis of lung cancer cells by activating the PI3K/Akt pathway. Med Oncol 32: 66, 2015.

16. Zhang L, Yin G, Yan D, Wei Y, Ma C, Huang Z, Liao X, Yao Y, Chen $X$ and Hao B: In vitro screening of ovarian tumor specific peptides from a phage display peptide library. Biotechnol Lett 33: 1729-1735, 2011. 